\title{
From Algorithm to Agent
}

\author{
Krzysztof Cetnarowicz \\ AGH University of Science and Technology, Krakow, Poland \\ cetnar@agh.edu.pl
}

\begin{abstract}
Although the notion of an agent has been used in computer science for a dozens of years now it is still not very well defined. It seems that there is a lack of formal definition of such concepts as an "object" and an "agent". It makes difficult formal analysis of algorithms developed with their use. We should find more formal description that has connection with the basic definition of the algorithm.

In the paper we will propose an approach that may help to develop more formal definitions of an agent and an object with the use of algorithm concept. Starting from the notion of the algorithm and using the observation that complex algorithm should be developed with the use of its decomposition we propose some ideas how we can consider such notions as object and agent.

Proposed approach takes into consideration the necessity of the autonomy and of an agent and object and the problems of the interactions between them and suggest the resolution of the problems by communication and observation process.

Presented concept of an object and an agent makes possible to find further more formal definitions of these notions and find the crucial properties of these concepts and the main difference between the notion of an object and the notion of an agent.
\end{abstract}

\section{Introduction}

Development of programming techniques and methods of software construction may be viewed as the development of algorithms working in a given environment ([14]). The process of creation of new more powerful algorithms resulted in definition of new elements that may be used to build them. Algorithms became more complex and more difficult to be developed. So, according to the principle: "divide and conquer" we will decompose the algorithm and the development process.

Although the notion of an agent has been used in computer science for dozens of years now it is still not very well defined. It seems that there is a lack of formal definition of such concepts as an "object" and an "agent". It makes formal analysis of algorithms developed with the use of concept of an object or an agent difficult ([10, [1], 12]).We should find better formal description that has connection with the base definition of the algorithm.

In the paper we will propose an approach that may help to develop more formal definitions of an agent and an object within the concept of an algorithm. 
Starting from the notion of an algorithm and using the observation that complex algorithm should be developed with the use of its decomposition we propose some ideas how we can consider such notions as object and agent. Proposed approach takes into consideration the necessity of the autonomy of an agent and an object and the problems of the interactions between them and suggest the resolution of the problems by communication and observation process. ([1], 2], [5], [7]).

Considering non-formal, based on intuition point of view of the concept of an algorithm we can say that an algorithm describes activity of an object or an agent in a given environment $([4]$, 8], 14]). These were programmers who noticed that current algorithms are hard to be developed because of their complexity, and the way to simplify the creation process is to use the previously mentioned method "divide and conquer". A number of approaches to divide (or rather decompose) an algorithm had been invented and applied. The idea of an object and then of an agent may be considered as the result of an attempt to enable the decomposition of an algorithm. Presented here approach to the notion of an object and an agent makes possible to find properties of these concepts and the difference between them.

\section{Algorithm and Decomposition}

We can consider the following definition of the algorithm $([13])$ :

$$
A l g=(U, F)
$$

where:

$$
U-\text { set }, \quad U \neq \emptyset, \quad F: U \rightarrow U
$$

Function $F$ is a partial function. It means that the domain of the function $F$ is a subset of the set $U$. Elements $u$ of the set $U$ are called states of the algorithm $A l g$.

Realization (execution) of the algorithm $A l g$ for a given initial state $u^{0}$ may be considered as a finite sequence [3]:

$$
u^{0}, u^{1}, \ldots u^{i}, u^{i+1}, \ldots u^{k}
$$

or infinite:

$$
u^{0}, u^{1}, \ldots u^{i}, u^{i+1}, \ldots
$$

such, that

$$
u^{i+1}=F\left(u^{i}\right)
$$

The sequence is finite when there is a final state $u^{k}$. There is a final state when $u^{k}$ belongs to the $\operatorname{Im}(F)$ and does not belong to the $\operatorname{Dom}(f)$. So, the final states of the algorithm $\mathrm{Alg}$ are elements of the set $U$ that do not belong to the domain of the function $F$.

To determine elements $u$ of the set $U$ is usually realized by characteristic properties of the elements $u$. We can consider elements of the set $U$ as n-tuples of characteristic parameters [6]:

$$
u^{k}=\left(x_{1}^{k}, x_{2}^{k}, \ldots, x_{m}^{k}\right)
$$


where every element $x_{j}^{i}$ determines a characteristic property $j$ of the element $u^{i}$. Instead of the set $U$ we use the set $X$ of n-tuples $\left(x_{1}^{k}, x_{2}^{k}, \ldots, x_{m}^{k}\right)$.

When we consider the function $F$ :

$$
F\left(u^{k}\right)=\left(u_{1}^{k+1}\right)
$$

and using the following expressions:

$$
u^{k}=\left(x_{1}^{k}, x_{2}^{k}, \ldots, x_{m}^{k}\right), \quad u^{k+1}=\left(x_{1}^{k+1}, x_{2}^{k+1}, \ldots, x_{m}^{k+1}\right)
$$

we can find that the function $F$ may be replaced by the partial function $f: X \rightarrow$ $X$, which operates on the mentioned above characteristic properties:

$$
f\left(x_{1}^{k}, x_{2}^{k}, \ldots, x_{m}^{k}\right)=\left(x_{1}^{k+1}, x_{2}^{k+1}, \ldots, x_{m}^{k+1}\right)
$$

In practical applications there is a great number of characteristic properties that define the states of the algorithm and the function $f$ is complex. Consequently, it is difficult to study and develop the algorithm. In such a case the best way is to decompose the the algorithm.

The decomposition of the algorithm $A l g$ concerns the set of characteristic properties $\left(x_{1}^{i}, x_{2}^{i}, \ldots, x_{m}^{i}\right)$, and the function $f$. As the result of such decomposition we have to obtain the decomposition of the complex algorithm Alg into a number of partial algorithms $A l g_{1}, A l g_{2}, \ldots A l g_{n}$.

The main goal of the decomposition is to enable the separate development of every partial algorithm. Then the complex algorithm is built as composition of the partial algorithms. Let us consider the algorithm $A l g=(X, f)$ defined by the set of states (or characteristic properties), and the function $f$ realizing the evolution of the algorithm. So we have:

$$
A l g=(X, f), \quad f: X \rightarrow X .
$$

Let the algorithm $A l g$ is decomposed into partial algorithms what means that instead the algorithm $A l g$ we consider a number of partial algorithms. To simplify our analysis we can consider that we have two partial algorithms $A l g_{r}$ and $A l g_{s}$. Using the partial algorithms $A l g_{r}$ and $A l g_{s}$ we may obtain the same results as the with the use of the algorithm $\mathrm{Alg}$.

The decomposition may concern the function $f$ and/or the set $X$. Let us consider as a first approach only the decomposition of the function $f$. The function $f$ may be decomposed into two partial functions $f_{r}$ and $f_{s}$ by the following way:

$$
\begin{array}{cc}
f=f_{r} \cup f_{s}, & f_{r} \cap f_{s}=\emptyset \\
f_{r}: X \rightarrow X, & A l g_{r}=\left(X, f_{r}\right) \\
f_{s}: X \rightarrow X, & A l g_{s}=\left(X, f_{s}\right)
\end{array}
$$

The successive application of functions $f_{r}$ and $f_{s}$ enables to transform the starting state of the algorithm to the final one that represents a needed solution. But the decomposition of only the function $f$ is not sufficient to enable the separate 
a)

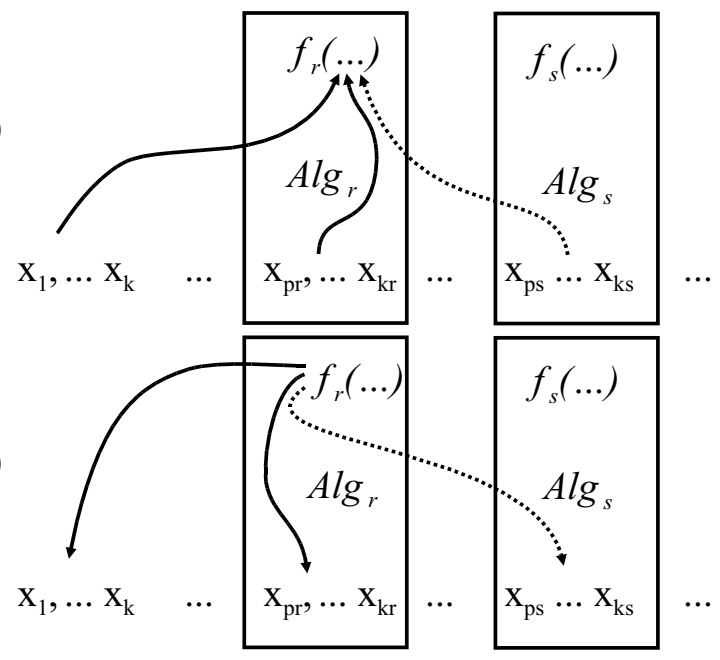

Fig. 1. Schema of the decomposition of characteristic properties

development of partial algorithms. We have to decompose the set $X$ of the algorithm $\mathrm{Alg}$. Let us consider following sets of properties of the algorithms: $X_{r}-$ representing properties of the algorithm $A l g_{r}, X_{s}$ - properties of the algorithm $A l g_{s}$ and the set $X_{0}$ describing resources of the environment that are accessible for every partial algorithm. The set $X$ is decomposed by the following way:

$$
X=X_{0} \times X_{r} \times X_{s}
$$

The set $X_{r}\left(X_{s}\right.$ - respectively) represents properties of the algorithm $A l g_{r}\left(A l g_{s}\right.$ - respectively) and for the sake of separate development of the algorithms $A l g_{r}$ and $A l g_{s}$ we have to enclose hermetically the properties of every algorithm. As a result of that the algorithm $A l g_{r}$ has no more access to the parameters $X_{s}$ and vice versa (dash arrow on the fig. 1).

We can consider two solutions proposed for this problem. One basing on the communication paradigm that leads us to the object concept, and the next one using operation of the observation that gives us the concept on agent.

\section{Decomposition of an Algorithm Using the Concept of an "Object"}

We can consider the decomposition of the set of characteristic properties. Every partial algorithm has its own subset of characteristic properties. A given partial algorithm $r\left(A l g_{r}\right)$ is defined by the function $f_{r}$ and the set of characteristic properties: $\left\{x_{p_{r}}, x_{p_{r}+1}, \ldots, x_{k_{r}}\right\}$ that is a subset of the global set of characteristic properties: $\left\{x_{1}, x_{2}, \ldots, x_{m}\right\}$. The characteristic properties of one partial algorithm 
(for instance $\left.A l g_{r}\right)$ may be used by the function of this algorithm $\left(f_{r}\right)$ and are not accessible to another algorithm (for instance $A l g_{s}$ ) what means that they may not be used by the function $f_{s}$ (fig. 1). Such consideration brought us to the concept of an "object" [13].

We can say that the partial algorithm $A l g_{r}$ may be considered as an object $O b j_{r}$, the state of the object is described by parameters $\left\{x_{p_{r}}, x_{p_{r}+1}, \ldots, x_{k_{r}}\right\}$ and the evolution of the state of this object is realized by the function $f_{r}^{o}$.

Considering proposed concept of an object we can notice that the lack of the access by the algorithm of a given object $O b j_{r}$ (by its function $f_{r}^{o}$ ) to the parameters $\left(\left\{x_{p_{s}}, x_{p_{s}+1}, \ldots, x_{k_{s}}\right\}\right)$ of the object $O b j_{s}$ is a too big restriction. To resolve the accessibility restriction problem a number of methods of access (under control) to the parameters of a given object has been proposed. The object $O b j_{s}$ may make its own parameters accessible for the function $f o_{r}$ of the object $O b j_{r}$ (fig. 2).

During the development we introduce the concept of the object tools of controlled access to the internal parameters of another object. So, a given object $O b j_{r}$ (its function $f_{r}^{o}$ ) has accessible its own parameters $\left\{x_{p_{r}}, x_{p_{r}+1}, \ldots, x_{k_{r}}\right\}$ called internal parameters of the object $O b j_{r}$, and in a limited way the parameters of other objects (for example to the parameters $\left\{x_{p_{s}}, x_{p_{s}+1}, \ldots, x_{k_{s}}\right\}$ of the object $O b j_{s}$. Obviously the object has access to the global parameters that are accessible to any object. There are tools to give control (restrictions) of the access to the internal parameters of an object. One of the most elegant tools is the use of methods defined in the body of the object to which internal parameters the access is realized. We may consider methods are tools for a particular communication between objects.

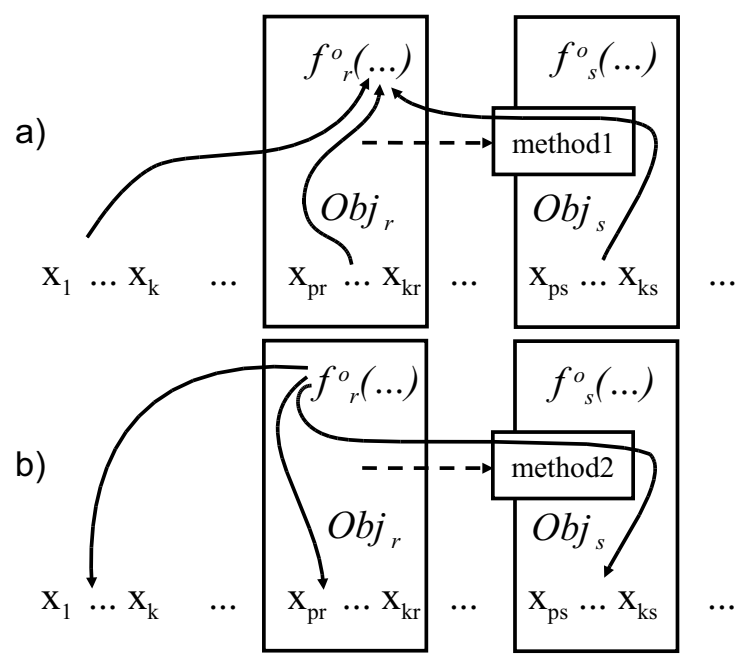

Fig. 2. Schema of the access of the object (partial algorithm) $O b j_{r}$ to the parameters of the object $O b j_{s}$ 
The scenario of the access of one object $\left(O b j_{r}\right)$ to parameters of another object $\left(O b j_{s}\right)$ may be described as follow:

- Function $f_{r}^{o}$ realizing execution of the object $O b j_{r}$ needs the following information: the value of its own parameters of the object $O b j_{r}$, global parameters and the value of some parameters of the object $O b j_{s}$.

- The object $O b j_{r}$ to get access to the parameters of the object $O b j_{s}$ put in execution (by calling) a properly selected method of the object $O b j_{s}$ and due to the execution of this method the object $O b j_{r}$ obtain information about parameters of the object $O b j_{s}$. The access is controlled by the object $O b j_{s}$ by the algorithm of its method and the access may be realized, partially realized or forbidden.

We can consider the function $f_{r}^{o}$ as following:

$$
\begin{aligned}
& \left(x_{1}^{\prime}, \ldots, x_{k}^{\prime}, x_{p_{r}}^{\prime}, \ldots, x_{k_{r}}^{\prime}, x_{p_{s}}^{\prime}, \ldots, x_{k_{s}}^{\prime}\right) \\
& =f_{r}^{o}\left(x_{1}, \ldots, x_{k}, x_{p_{r}}, \ldots, x_{k_{r}}, \operatorname{method} 1\left(x_{p_{s}}, \ldots, x_{k_{s}}\right)\right) .
\end{aligned}
$$

- When the object $O b j_{r}$ has all the necessary information it can realize evolution of the state of the algorithm using its own function $f_{r}^{o}$. In the result of the execution the parameters of the algorithm may be modified. Object $O b j_{r}$ may modify without restrictions its own parameters $\left(x_{p_{r}}, x_{p_{r}+1}, \ldots, x_{k_{r}}\right)$ and global parameters. But sometimes as the result of application of the function $f_{r}^{o}$ the modification of internal parameters of another $\left(O b j_{s}\right)$ is necessary. For this purpose the methods of the object $O b j_{s}$ may be used. Object $O b j_{r}$ using the properly chosen method of the object $O b j_{s}$ may change value of internal parameters of that object ( for instance: method $2\left(x_{p_{s}}^{\prime}, \ldots, x_{k_{s}}^{\prime}\right)$ (fig. 2) ). Obviously this modification is controlled by the object $O b j_{s}$ by algorithms of methods used.

The presented scenario of the communication between objects causes independency of an object to be considerably restricted. For instance in presented scenarios the object $O b j_{s}$ may not give access to its internal parameters for the object $O b j_{r}$ what may block algorithm of the object $O b j_{r}$. We can say that autonomy of the object $O b j_{r}$ is depended (under restriction) on the object $O b j_{s}$ (fig. 2).

Using the proposed concept of the object with tools of communication by the "call of method" we can decompose a given complex algorithm into partial algorithms represented by objects ([9]. Cooperation of this objects makes possible the execution of the complex algorithm as a whole. The realization of the complex algorithm is the result of the successive application of the functions $f_{r}^{o}$ and $f_{s}^{o}$ in a properly defined order (cooperation of the objects $O b j_{r}$ and $O b j_{s}$ ).

\section{Decomposition of an Algorithm with the Concept of an "Agent"}

The loss of independency (or autonomy) of partial algorithms realized as objects is the price paid for the cooperation of objects in the system realized by the object oriented approach. 


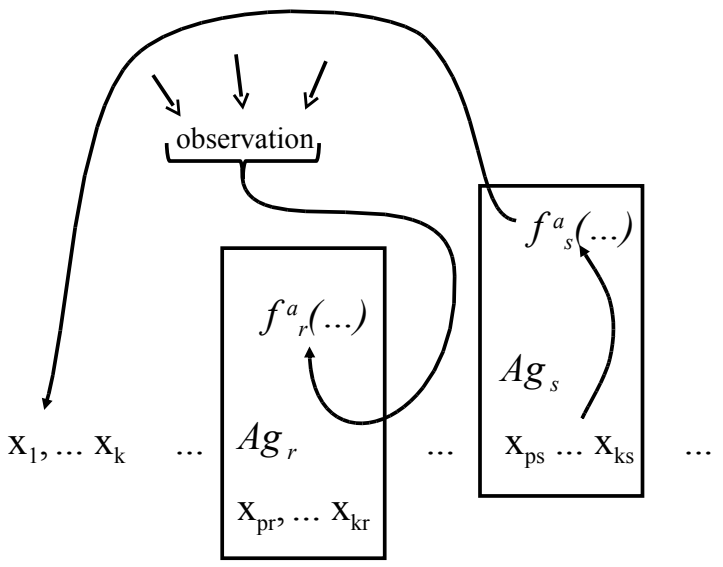

Fig. 3. Schema of execution of the algorithms of objects with the use of observation of behavior of other objects. It is a principle of the concept of an agent.

We can try to find a solution where partial algorithms are realized as entities more independent than objects. It is possible due to the observation operation. Using the observation operation one algorithm may observe behavior of another one. It leads us to the concept of an agent. The way of the action of a given agent may be illustrated by the following example:

- Function realizing algorithm of the agent $A g_{r}$ needs its own parameters, global data, and parameters of the agent $A g_{s}$.

- Therefore the agent $A g_{r}$ observes behavior of the agent $A g_{s}$. It means the agent $A g_{r}$ follows changes in the environment (global data) that are caused by the actions of the agent $A g_{s}$. Due to this observation agent $A g_{r}$ may deduce the state of parameters of the agent $A g_{s}$. This observation and deduction is an independent process of intentions of the agent $A g_{s}$, although the received data do not give the full information about the internal parameters of the agent $A g_{s}$. But information obtained may be sufficient for the agent $A g_{r}$ to continue its actions.

- Agent $A g_{r}$ being in possession of necessary information, and using its function $f_{r}$ may modify its own parameters and state (global parameters) of the environment. These changes are realized usually as an execution of an event in the environment. Agent $A g_{r}$ has no possibilities to change directly the internal data of the agent $A g_{s}$ but by the means of modification of the state of the environment it have impact on the state of other agents. Agent $A g_{s}$ observes the changes in the environment caused by the agent $A g_{r}$. Due to this changes agent $A g_{s}$ modifies its own parameters.

Agent observing environment (global data) and behavior of other agents may realize its actions (fig. 3). Presented concept of an agent gives considerable 


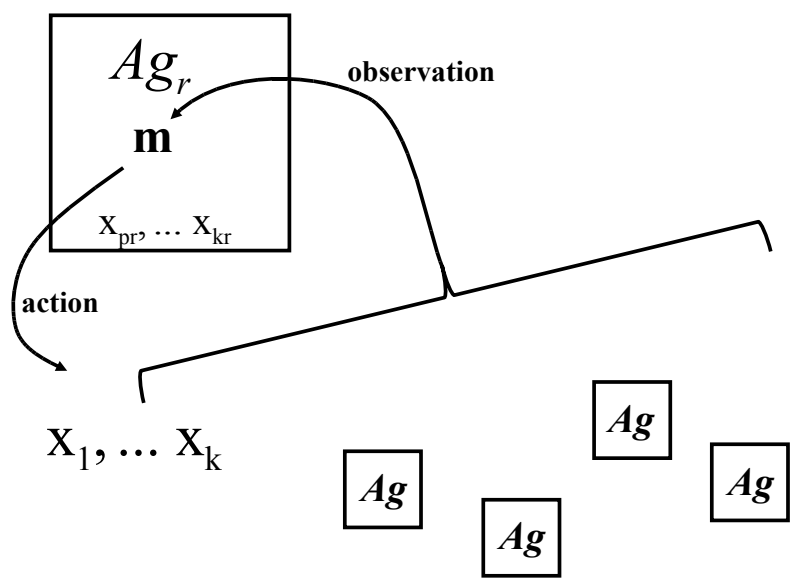

Fig. 4. Schema of an agent acting in the environment with the use of its model

augmentation of independence of an agent and possibility of interaction between agents. That is the crucial difference between the concepts of the object and the agent.

Resuming we can say that the source of information for the agent is the state of the global data (state of the environment, or resources of the environment represented by $\left.\left(x_{1}, \ldots, x_{k},\right)\right)$, local data of the agent (state of the agent $A g_{r}$ represented by $\left.\left(x_{p r}, \ldots, x_{k r},\right)\right)$ and observed behavior of other agents (for instance $\left.A g_{s}\right)$. This information agent may acquire by observation of the environment(fig. 41).

The algorithm is created as the environment containing resources (represented by global data) and agents that remain and act in the environment.

Not all information acquired by the observation of the environment is necessary for a given agent to be able to act in the environment. To select the only necessary information agent may use a method of modeling the environment. Due to the operation of observation a given agent builds in its mind a model of the environment. Using this model $m$ agent may select the necessary information, and then use it to realize its action in the environment. This selection is realized by the adopted abstraction level of the model construction and its manipulation.

Using presented idea we can consider the following scenario of action by a given agent that is in a given environment:

- Agent using the operation of observation acquires information from the environment and builds a model $(m)$ of the environment in its mind. The model is created with adopted by the agent abstraction level - what means that in the model only some characteristic properties of the environment are memorized (only information necessary for agents's actions).

- Agent plans actions in the environment using the analysis of created model $\mathrm{m}$.

- Agent carries out the planned actions realizing events in the environment. 
Agent $A g_{r}$ to observe the changes in the environment caused by the agent $A g_{s}$ has to memorize in the model $m$ not only actual state of the environment $\left(x_{1}^{i}, \ldots, x_{k}^{i},\right)$ but also , historical data" $\left(x_{1}^{i-1}, \ldots, x_{k}^{i-1},\right)$ describing the state of the environment before changes caused by the agent $A g_{s}$. That is the model $m$ of the agent that contains all data (including "historical data") necessary for the agent to act in the environment. The application of the model $m$ may enable the autonomy of the agent and realize cooperation among agents.

\section{Conclusion}

Presented approach to the description of decomposition of the algorithm helps to define the concept of the object and the agent.

The paper makes possible to explain reasons for introduction of the object and agent notions. It enables to determine the principal difference between an object and an agent and to establish their main properties.

It may be useful for development of Object Oriented Technologies and Agent Oriented Technologies to create complex multiagent systems.

\section{References}

1. Cetnarowicz, E., Cetnarowicz, K., Nawarecki, E.: The simulation of the behavoiur of the world of autonomous agents. In: Proc. of the XVII International Czech Poland - Slovak Colloqium - Workshop: Advanced Simulation of Systems, Zabrzech na Morave, Czech Republic, vol. 2, pp. 107-115 (1995) ISBN 80-901751-4-7

2. Cetnarowicz, K.: Problems of the evolutionary development of the multi-agent world. In: Proc. of the First International Workshop: Decentralized Multi-Agent Systems DIMAS 1995, Krakow, Poland, pp. 113-123 (1995) ISBN 83-86813-10-5

3. Cetnarowicz, K., Cetnarowicz, E.: Multi-agent decentralised system of medical help. In: Management and Control of Production and Logistics. IFIP, IFAC, IEEE Conference, Grenoble, France, 2000. ENSIEG, LAG Grenoble, France (2000)

4. Cetnarowicz, K., Dobrowolski, G., Ko@zlak, J.: Active agents cooperation in decentralized systems. In: Bubnicki, Z. (ed.) Proc. of the 12th Int. Conf. on Systems Science, vol. 1, pp. 57-62. Oficyna Wydawnicza Politechniki Wroc@lawskiej (1995) ISBN 83-7085-152-5

5. Cetnarowicz, K., Nawareck, E.: Système d'exploitation decentralisé realisé à l'aide de systèmes multi-agents (Operating System Realized with the Use of Multi-agent Systems). In: Proceedings, Troisième Journées Francophone sur l'Intelligence Artificielle Distribuée et les Systèmes Multiagents, St Baldoph, Savoie, Francja, pp. 311-322 (1995)

6. Crowley, J.L., Demazeau, Y.: Principles and techniques for sensor data fusion. In: Signal Processing, vol. 32, pp. 5-27. Elsevier Science Publishers B. V., Amsterdam (1993)

7. Nawarecki, E., Cetnarowicz, K.: A concept of the decentralized multi-agent rt system. In: Proc. of the International Conference Real Time 1995, Technical University of Ostrava VSB, Ostrava, Czech Republic, pp. 167-171 (1995) ISBN 80-901751-6-3 
8. Nawarecki, E., Cetnarowicz, K., Cetnarowicz, E., Dobrowolski, G.: Active agent idea applied to the decentralized intelligent systems development. In: Štefan, J. (ed.) Modeling and Simulation of Systems MOSIS 1994, Ostrava, pp. 64-71. House of Technology Ltd. (1994) ISBN 80-901229-8-1

9. Nicola, J., Coad, P.: Object-Oriented Programming. Prentice Hall, Inc., Englewood (1993)

10. Rao, A.S., Georgeff, M.P.: Modelling rational agents within a bdi architecture. In: Proc. of the Second International Conference on Principles of Knowledge Representation and Reasoning, KR 1991, Cambridge, MA, USA, pp. 473-484 (1991)

11. Shoham, Y.: Agent-oriented programming. Artificial Intelligence 60, 51-92 (1993)

12. Weiss, G.: Multiagent Systems. MIT Press, Cambridge (1999)

13. Winkowski, J.: Programowanie symulacji procesow (Programming of Simulation Process). Wydawnictwo Naukowo-Techniczne, Warszawa (1974)

14. Wirth, N.: Algorithms+Data Structures = Programs. Prentice-Hall Series in Automatic Computation (1976) 